\title{
Expression of GLS1 in intrahepatic cholangiocarcinoma and its clinical significance
}

\author{
JUN CAO $^{1}$, CHI ZHANG ${ }^{2}$, GUO-QING JIANG ${ }^{2}$, SHENG-JIE JIN ${ }^{2}$, ZHI-HUI GAO $^{2}$, QIAN WANG ${ }^{2}$, \\ DE-CAI YU ${ }^{3}$, AI-WU KE ${ }^{4}$, YI-QUN FAN ${ }^{5}$, DA-WEI LI ${ }^{5}$, AO-QING WANG ${ }^{2}$ and DOU-SHENG BAI ${ }^{2}$
}

\author{
${ }^{1}$ Department of Hepatobiliary and Pancreatic Surgery, The Second Affiliated Hospital of Xiangya School of Medicine, \\ Central South University, Changsha, Hunan $410008 ;{ }^{2}$ Department of Hepatobiliary Surgery, Clinical Medical College, \\ Yangzhou University, Yangzhou, Jiangsu 225000; ${ }^{3}$ Department of Hepatobiliary Surgery, The Affiliated Drum Tower Hospital, \\ Medical School of Nanjing University, Nanjing, Jiangsu 210093; ${ }^{4}$ Liver Cancer Institute, Ministry of Education, \\ Zhongshan Hospital, Fudan University, Key Laboratory of Carcinogenesis and Cancer Invasion (Fudan University), \\ Shanghai 200032; ${ }^{5}$ Department of Surgery, Dalian Medical University, Dalian, Liaoning 116044, P.R. China
}

Received November 19, 2018; Accepted May 10, 2019

DOI: $10.3892 / \mathrm{mmr} .2019 .10399$

\begin{abstract}
Kidney-type glutaminase (GLS1) plays a significant role in tumor metabolism. Our recent studies demonstrated that GLS1 was aberrantly expressed in hepatocellular carcinoma (HCC) and facilitated tumor progression. However, the roles of GLS1 in intrahepatic cholangiocarcinoma (ICC) remain largely unknown. Thus, the aim of this study was to evaluate the expression and clinical significance of GLS1 in ICC. For this purpose, combined data from the Oncomine database with those of immunohistochemistry were used to determine the expression levels of GLS1 in cancerous and non-cancerous tissues. Second, a wound-healing assay and Transwell assay were used to observe the effects of the knockdown and overexpression of GLS1 on the invasion and migration of ICC cells. We examined the associations between the expression of GLS1 and epithelial-mesenchymal transition (EMT)-related markers by western blot analysis. Finally, we examined the associations between GLS1 levels and clinicopathological factors or patient prognosis. The results revealed that GLS1 was overexpressed in different digestive system tumors, including ICC, and that GLS1 expression in ICC tissue was higher than that in peritumoral tissue. The overexpression of GLS1 in RBE cells induced metastasis and invasion. Moreover, the EMT-related markers, E-cadherin and Vimentin, were regulated by GLS1 in ICC cells. By contrast, the knockdown of GLS1 expression in QBC939 cells yielded opposite results. Clinically, a high
\end{abstract}

Correspondence to: Professor Dou-Sheng Bai, Department of Hepatobiliary Surgery, Clinical Medical College, Yangzhou University, 98 West Nantong Road, Yangzhou, Jiangsu 225000, P.R. China

E-mail: drbaidousheng@126.com

Key words: intrahepatic cholangiocarcinoma, kidney-type glutaminase, metastasis, invasion, epithelial-mesenchymal transition expression of GLS1 in ICC samples negatively correlated with E-cadherin expression and positively correlated with Vimentin expression. GLS1 protein expression was associated with tumor differentiation $(\mathrm{P}=0.001)$ and lymphatic metastasis $(\mathrm{P}=0.029)$. Importantly, patients with a high GLS1 expression had a poorer overall survival (OS) and a shorter time to recurrence than patients with a low GLS1 expression. Multivariate analysis indicated that GLS1 expression was an independent prognostic indicator. On the whole, the findings of this study demonstrated that GLS1 is an independent prognostic biomarker of ICC. GLS1 facilitates ICC progression and may thus prove to be a therapeutic target in ICC.

\section{Introduction}

Intrahepatic cholangiocarcinoma (ICC) is the second most common intrahepatic primary tumor after hepatocellular carcinoma (HCC) (1). Despite improvements in surgical techniques, the resectability and curability of ICC remain unsatisfactory (2). Currently, the main treatment option for ICC is curative resection; however, the prognosis of patients with ICC remains poor due to the high frequency of recurrence and metastasis following surgical resection (3-5). Thus, the elucidation of the underlying mechanisms involved in ICC hepatocarcinogenesis is of utmost importance.

Glutaminase is an amidohydrolase that catalyzes the first step in the glutaminolysis of glutamine to glutamate. Glutaminase exists as two isoforms, GLS1 and GLS2, which were originally identified as kidney and liver glutaminases, respectively. The majority of cancer types, including ICC, require a constant supply of glutamine to support tumor progression and cell proliferation (6). An increasing number of studies have indicated that an aberrant expression level of GLS1 is associated with cancer invasion and metastasis in HCC, breast cancer cell lines and colorectal cancer (7-9). These findings indicate that GLS1 is a key mediator of tumor migration and invasion. It has been demonstrated that epithelial-mesenchymal transition (EMT) is a critical cause of invasion and migration 
in a number of types of cancer of epithelial origin (10). EMT involves profound phenotypic alterations, including the loss of epithelial cell polarity following reductions in the levels of epithelial proteins, such as E-cadherin and increases in the levels of mesenchymal proteins that increase mesenchymal proliferation and invasion (11). Recently, a study reported that GLS1 metabolism is possibly involved in the activation of TGF- $\beta$ /Wnt signaling and the induction of EMT (12). Thus, GLS1 may well become a novel target for the treatment of malignant tumor cells undergoing EMT-driven invasion and mobility. However, the specific association between GLS1 and EMT-related markers in ICC cells remains unclear.

In this study, we examined the expression of GLS1 in ICC and investigated the role and mechanisms of action of GLS1 in ICC cell invasion and migration. In addition, clinical characteristics, such as overall survival (OS) and the cumulative recurrence rate were also assessed.

\section{Materials and methods}

Patients and samples. In this study, a tissue microarray was used containing 138 paired paraffin-embedded ICC tissue samples and corresponding peritumoral tissues samples obtained from patients who had undergone hepatic resection at Zhongshan Hospital, Fudan University from 2007 to 2012. The use of these tissue specimens was approved by the Zhongshan Hospital Research Ethics Committee and written consent was obtained from the patients. The detailed clinicopathological characteristics of the patients are presented in Table I.

Cells and cell culture. Three ICC cell lines, QBC939, HCCC-9810 and RBE, were obtained from the Institute of Biochemistry and Cell Biology of the Chinese Academy of Sciences and maintained in RPMI-1640 medium supplemented with $10 \%$ fetal bovine serum, penicillin $(100 \mathrm{U} / \mathrm{ml})$ and streptomycin $(100 \mu \mathrm{g} / \mathrm{ml})$ (all Gibco; Thermo Fisher Scientific) under $95 \%$ air and $5 \% \mathrm{CO}_{2}$ at $37^{\circ} \mathrm{C}$.

Tissue microarrays (TMAs), immunohistochemistry and hematoxylin-eosin $(H \& E)$ staining. A tissue microarray that included 138 ICC tissue samples with corresponding adjacent tissue samples was constructed by Shanghai Outdo Biotech Co., Ltd. (13). Briefly, 4- $\mu \mathrm{m}$ sections were obtained from the TMA blocks using a microtome Shanghai Outdo Biotech Co., Ltd. The $4-\mu \mathrm{m}$ sections on the TMAs were dried at $60^{\circ} \mathrm{C}$ for $4 \mathrm{~h}$, de-paraffinized in xylene I for $10 \mathrm{~min}$, de-paraffinized in xylene II for another $10 \mathrm{~min}$, and then rehydrated in graded ethanol (100\% ethanol for $5 \mathrm{~min}, 90 \%$ ethanol for $5 \mathrm{~min}, 80 \%$ ethanol for $5 \mathrm{~min}$ and $70 \%$ ethanol for $5 \mathrm{~min}$ ). Ethanol was subsequently removed by washing with phosphate-buffered saline (PBS) for $15 \mathrm{~min}$. For immunohistochemistry, the sections were incubated in $3 \% \mathrm{H}_{2} \mathrm{O}_{2}$ at room temperature for 20 min to block endogenous peroxidases. The TMAs were then microwaved in EDTA [1:500; pH 8.0; Absin (Shanghai) Biotechnology Co., Ltd.] for $15 \mathrm{~min}$ for antigen retrieval and subsequently incubated with $1 \%$ bovine serum albumin [1:500; pH 8.0; Absin (Shanghai) Biotechnology Co., Ltd.]. Subsequently, TMAs were incubated with the following rabbit anti-human primary antibodies for $12 \mathrm{~h}$ at $4^{\circ} \mathrm{C}$ : Anti-GLS1 (1:200; cat. no. ab93434; Abcam), anti-E-cadherin
(1:200, cat. no. 24E10; Cell Signaling Technology, Inc.) and anti-Vimentin (1:150; cat. no. D21H3; Cell Signaling Technology, Inc.). Subsequently, TMAs were incubated with horseradish peroxidase-labeled secondary antibodies [1:1,000; cat. no. abs957; Absin (Shanghai) Biotechnology Co., Ltd.] for $2 \mathrm{~h}$ at $37^{\circ} \mathrm{C}$. DAB [1:500; Absin (Shanghai) Biotechnology Co., Ltd.] was used as a detection reagent and was incubated with the TMAs for $1.5 \mathrm{~min}$, after which, the samples were counterstained with hematoxylin for $2 \mathrm{~min}$ at room temperature, and were subsequently dehydrated in a gradient series of ethanol. Images of the sections were subsequently captured under a light microscope (Olympus BX-51; Olympus Corporation). All specimens were reviewed and scored by investigators blinded to the clinical characteristics of the patients. The expression of GLS1, Vimentin and E-cadherin was scored as follows: 0, no staining; 1, weak staining; 2 , moderate staining; and 3 , strong staining. The percentage of positively stained cells was scored as follows: $0,0-10 \% ; 1,1-25 \% ; 2,26-50 \% ; 3,51-75 \%$; and $4,>75 \%$. A final score of $0-2$ was considered as negative expression, whereas a score of 3-12 was considered as positive expression.

For H\&E staining, sections were stained with hematoxylin solution $(0.2 \%)$ for $4 \mathrm{~min}$, followed by eosin solution $(0.5 \%)$ for $90 \mathrm{sec}$ at room temperature.

Western blot analysis. Cell and tissue proteins were extracted using radioimmunoprecipitation assay buffer (cat. no. P0013C; Beyotime Institute of Biotechnology), and the protein concentration was measured using an enhanced Bicinchoninic Acid Protein Assay kit (cat. no. P0010; Beyotime Institute of Biotechnology). Proteins were loaded at $20 \mu \mathrm{g} / \mathrm{lane}$, separated by $10 \%$ SDS-PAGE (cat. no. P0012A; Beyotime Institute of Biotechnology) and were transferred to polyvinylidene fluoride membranes (EMD Millipore) for western blot analysis. Subsequently, the membranes were blocked with TBS containing $0.1 \%$ Tween 20 and 5\% non-fat milk for $2 \mathrm{~h}$ at room temperature, and were subsequently incubated with the following rabbit primary antibodies at $4^{\circ} \mathrm{C}$ for $12 \mathrm{~h}$ : Anti-GLS1 (1:1,000; cat. no. ab93434; Abcam), anti-E-cadherin (1:1,000; cat. no. 24E10; Cell Signaling Technology, Inc.), anti-Vimentin (1:500; cat. no. D21H3; Cell Signaling Technology, Inc.) and anti-GAPDH (1:1,000; cat. no. D16H11; Cell Signaling Technology, Inc.). The membranes were then rinsed and incubated with secondary antibody (1:5,000; cat. no. A0208; Beyotime Institute of Biotechnology) at room temperature for $2 \mathrm{~h}$. Densitometric analysis using an enhanced chemiluminescence system (EMD Millipore) and ImageJ software (version 1.49; National Institutes of Health) was performed to detect protein expression.

Regulation of GLS1 by siRNA and pcDNA transfection. siRNAs and a pcDNA plasmid that can regulate the human GLS1 gene were obtained from Shanghai Genomeditech Co. Two siRNAs were designed to silence GLS1, and the cells were randomly divided into the siRNA1-transfected cell group; siRNA2-transfected cell group; the negative control (NC) group, which was transfected with non-targeting siRNA; and the mock group, which consisted of untransfected cells. A pc-DNA3.1 plasmid was designed to induce overexpression of GLS1, and the cells were divided into the 
Table I. Associations between GLS1 with clinicopathologic characteristics of the 138 patients with ICC.

\begin{tabular}{|c|c|c|c|}
\hline \multirow[b]{2}{*}{ Characteristic } & \multicolumn{2}{|c|}{ No. of patients } & \multirow[b]{2}{*}{ P-value } \\
\hline & GLS $1^{\text {low }}$ & GLS1 $1^{\text {high }}$ & \\
\hline
\end{tabular}

Sex

Male

Female

25

38

Age, years

$\geq 53$

$<53$

HBsAg

Positive

Negative

Child-Pugh score

$$
\text { A }
$$$$
\text { B }
$$

Serum CA 19-9, ng/Ml

$\geq 37$
$<37$
CEA
$\geq 3.4$
$<3.4$

Serum ALT, U/1

$\geq 75$

$<75$

Serum AFP, ng/ml

$\geq 20$

$<20$

GGT

$\geq 75$

$<75$

Cirrhosis

Yes

No

27

36

33

42

43

32

38

25

62

1

33

30

22

41

34

41

48

27

70

5

0.061

0.215

0.872

10

$\begin{array}{rr}9 & 10 \\ 54 & 65\end{array}$

65

0.871

0.485

39

36

29

34

24

39

Tumor size (diameter, $\mathrm{cm}$ )

$\geq 5$

49

14

Tumor number

Multiple

Solitary

60

Embolus

Yes

9

54

No

66

39

0.586

32

43

58

17

Capsulation

Yes

Lymphatic metastasis

Yes

No

0.090
Table I. Continued.

\begin{tabular}{lccc}
\hline & \multicolumn{2}{c}{ No. of patients } & \\
\cline { 2 - 3 } Characteristic & GLS1 $1^{\text {low }}$ & GLS1 $1^{\text {high }}$ & P-value \\
\hline Tumor differentiation & & & $\mathbf{0 . 0 0 1}$ \\
III/IV & 21 & 46 & \\
I/II & 42 & 29 & \\
\hline
\end{tabular}

Fisher's exact test was used to determine the P-values. GLS1 ${ }^{\text {high }}, \geq 50 \%$ staining; GLS1 $1^{\text {low }},<50 \%$ staining. Statistically significant values are shown in bold. AFP, $\alpha$-fetoprotein; ALT, alanine aminotransferase; CA19-9, carbohydrate antigen 19-9; HBsag, hepatitis B surface antigen; GGT, $\gamma$-glutamyl transferase; ICC, intrahepatic cholangiocarcinoma; GLS1, kidney-type glutaminase.

pc-DNA3.1-GLS1-transfected cell group; the NC group, which was transfected with an empty pc-DNA3.1 vector; and the mock group, which consisted of untransfected cells. The siRNA sequences were as follows: siRNA1, upstream 5'-CCA GGUUGAAAGAGUGUAUTT-3', downstream 5'-AUACAC UCUUUCAACCUGGTT-3'; siRNA2, upstream 5'-CCCUGA AGCAGUUCGAAAUTT-3', downstream 5' AUUUCGAAC UGCUUCAGGGTT-3'; NC, upstream 5'-UUCUCCGAA CGUGUCACGUTT-3', downstream 5'-ACGUGACACGUU CGGAGAATT-3'. Briefly, cells were seeded at a density of $1 \times 10^{6}$ cells/well in 6 -well plates and were incubated until $70 \%$ confluence was reached. siRNAs and pc-DNA3.1 plasmids were transfected into the QBC939 and RBE cells using Lipofectamine ${ }^{\circledR} 2000$ (Invitrogen; Thermo Fisher Scientific, Inc.) according to the manufacturer's instructions. After $72 \mathrm{~h}$, the cells were harvested for total protein extraction and examined by western blot analysis.

Cell migration assays. Cell migration was assessed using a wound-healing assay. ICC cells were plated in a 6-well plate and were incubated until they reached $100 \%$ confluence. Following serum starvation for 1 day, cells were wounded with a $200-\mu 1$ plastic tip. Cells were washed three times with sterile PBS to remove the floating cells and were then incubated for $24 \mathrm{~h}$ at $37^{\circ} \mathrm{C}$ under $95 \%$ air and $5 \% \mathrm{CO}_{2}$. The same wound areas were observed and images were captured under a light microscope (Olympus IX71; Olympus Corporation) at 0 and $24 \mathrm{~h}$. The migratory abilities were quantified by measuring the percentage of the migration area of cells in the scratched regions, as follows: $0 \mathrm{~h}$ scratch area-24 $\mathrm{h}$ scratch area/0 h scratch area $\mathrm{x} 100 \%$.

Cell invasion assays. A Transwell assay was conducted to assess the invasive ability of cells in response to GLS1 overexpression or knockdown. The upper surface of the Transwell 0.252 filter used in the assay was coated with Matrigel. Cells $\left(1 \times 10^{5}\right)$ suspended in $150 \mu \mathrm{l}$ serum-free medium were added to the Transwell chamber (cat. no. 3413; Corning, Inc.), which was placed into a 24-well plate containing complete medium. After $24 \mathrm{~h}$ of incubation at $37^{\circ} \mathrm{C}$, the filter was extracted and cells on the upper surface of the filter were removed with cotton swabs. Cells on the underside of the Transwell filter were 

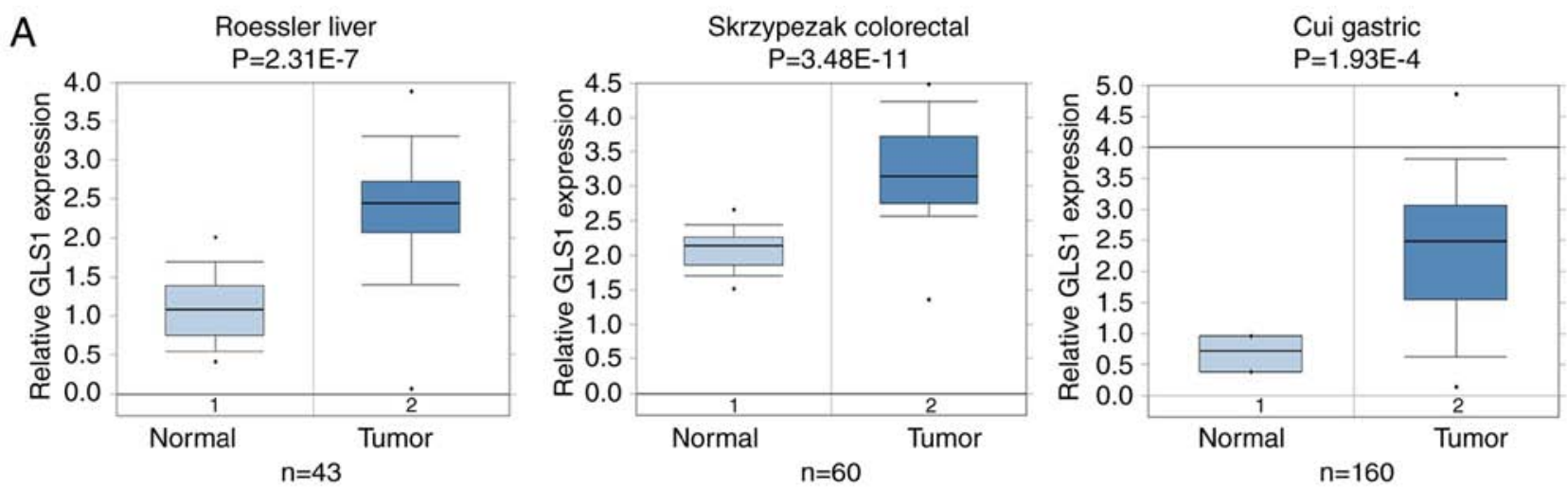

B
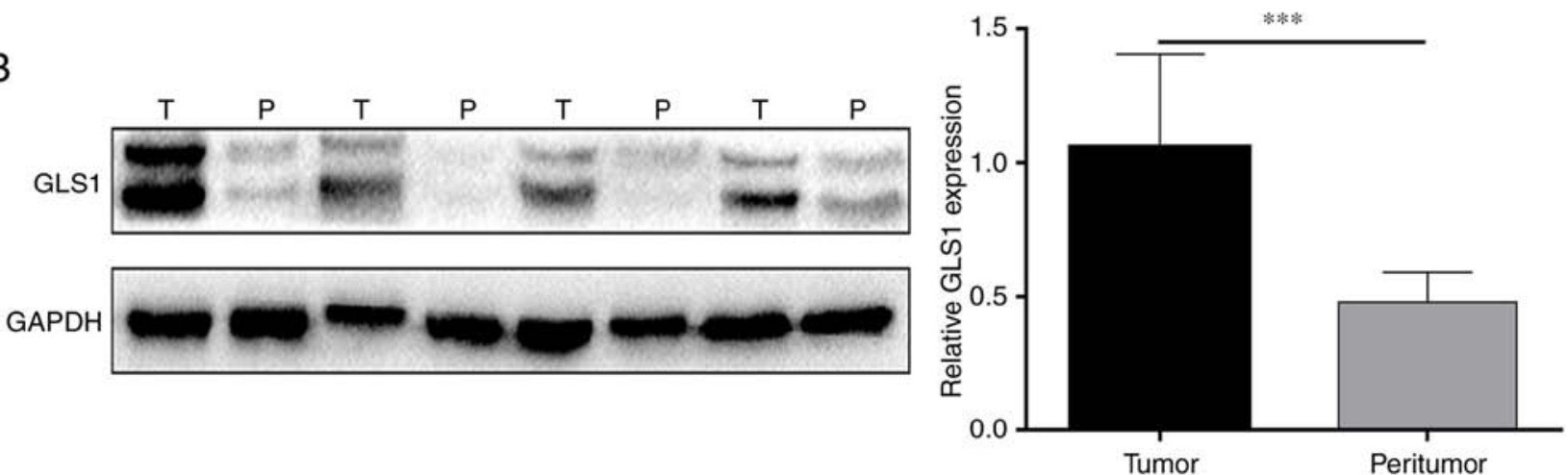

C
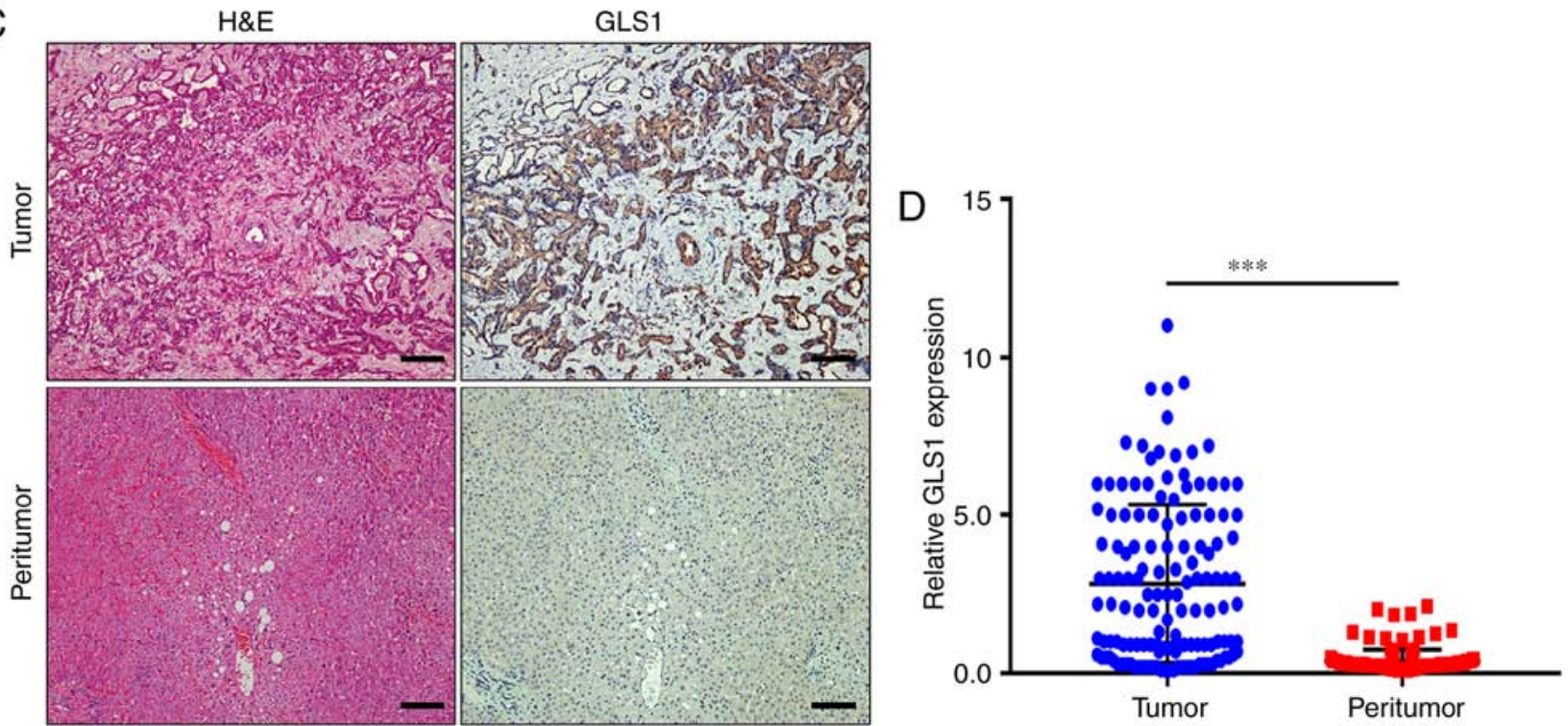

Figure 1. GLS1 expression is upregulated in primary ICC. (A) An Oncomine-based microarray database query of GLS1 gene expression in hepatocellular carcinoma, colorectal cancer and gastric cancer was performed. (B) The GLS1 protein level in 4 paired of human primary ICC tissue (T) and adjacent normal tissue (P) samples was determined by western blot analysis. (C and D) Immunohistochemistry was used to analyze the GLS1 expression in a TMA containing 138 samples of ICC patient tumor tissue and peritumoral tissue. Scale bars, $100 \mu \mathrm{m}$. "P<0.05, ${ }^{* * *} \mathrm{P}<0.001$. GLS1, kidney-type glutaminase; ICC, intrahepatic cholangiocarcinoma; TMA, tissue microarray.

fixed with $4 \%$ paraformaldehyde for $25 \mathrm{~min}$ and stained with $0.1 \%$ crystal violet for $15 \mathrm{~min}$ at $37^{\circ} \mathrm{C}$, after which, images were captured (Olympus IX71; Olympus Corporation) and the number of cells was quantified.

Statistical analysis. Statistical analyses were performed using SPSS 23.0 (IBM Corp.) and GraphPad Prism7 (GraphPad Software, Inc.) software. The Student's t-test was used to compare differences between groups. One-way analysis of variance (ANOVA) was used to compare differences among groups. Spearman's correlation analysis was used for the correlation analysis and Fisher's exact test was used to determine the association of GLS1 with ICC characteristics. OS and the cumulative recurrence rate were determined using Kaplan-Meier survival curves and the log-rank test. Independent prognostic factors were evaluated by with Cox proportional hazards model. A P-value $<0.05$ was considered to indicate a statistically significant difference. 
A

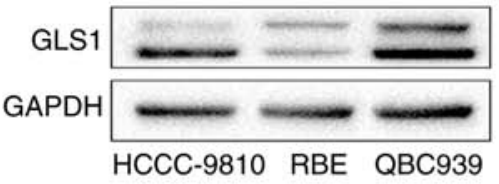

B
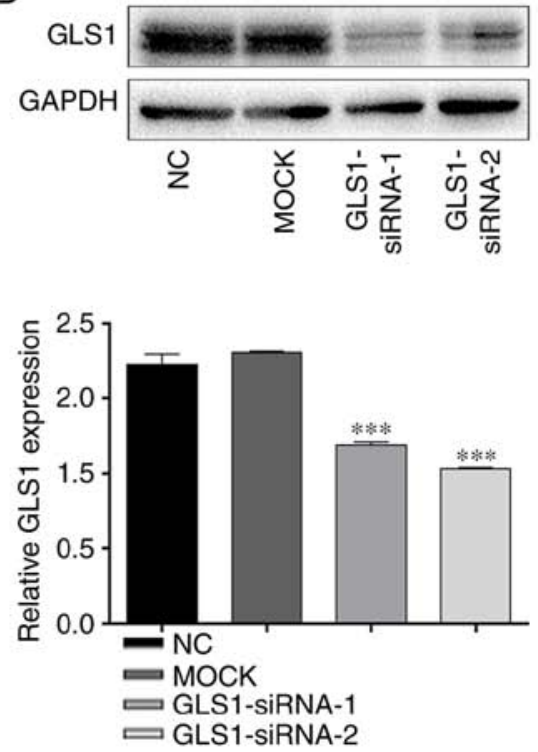

C
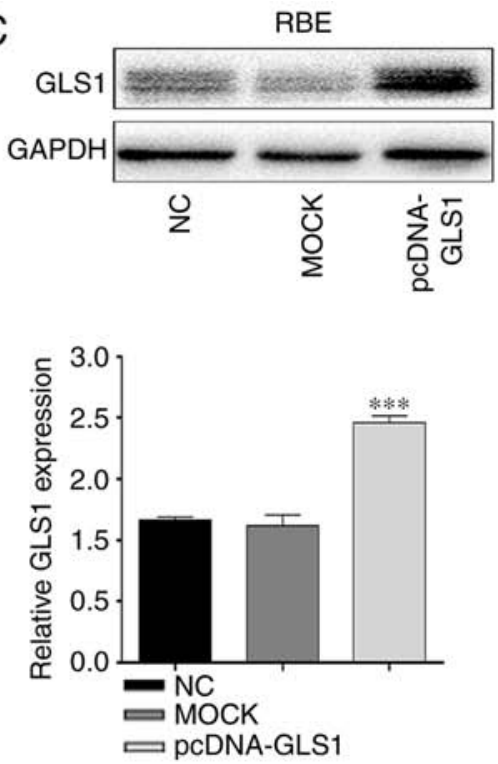

D
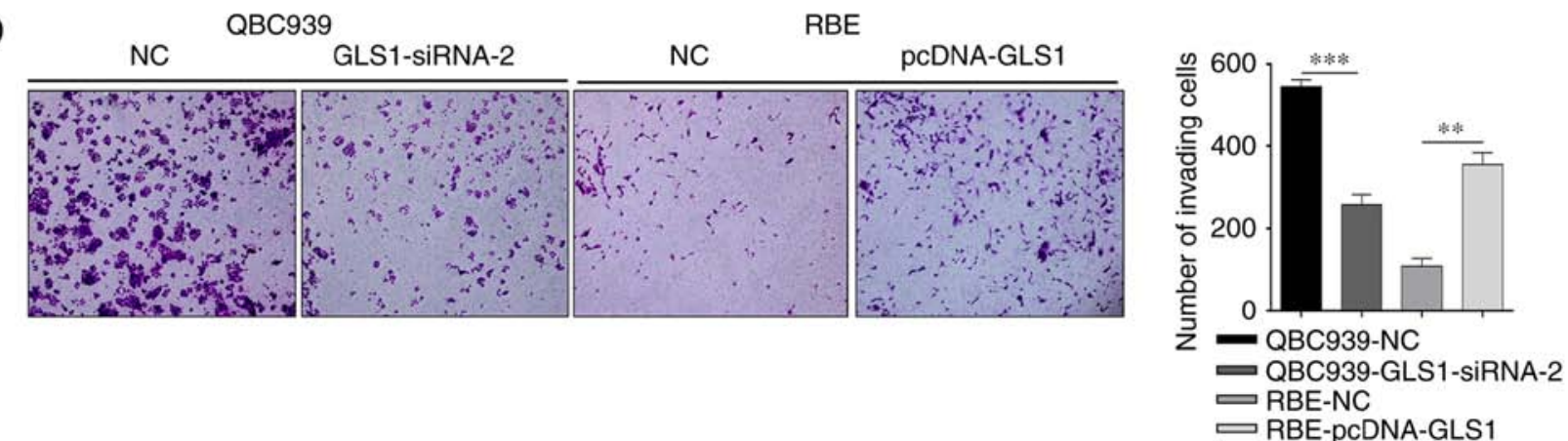

E

QBC939
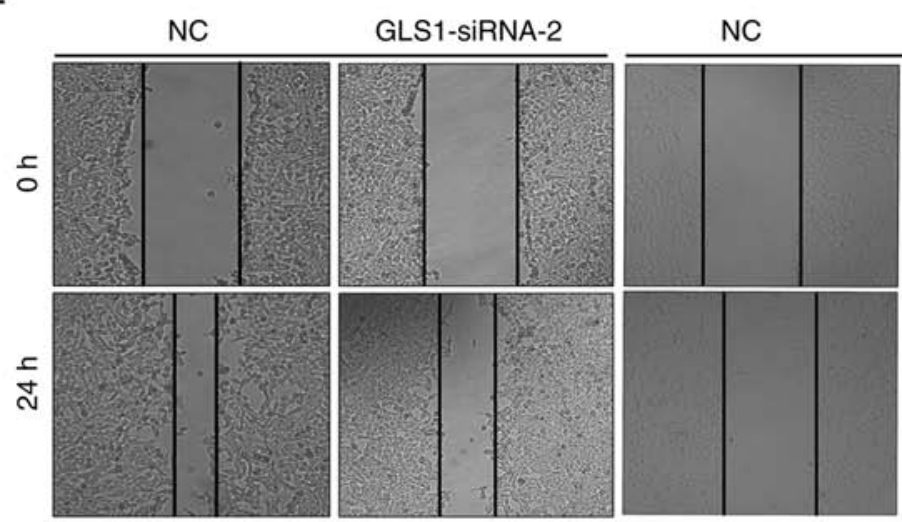

RBE

pcDNA-GLS1
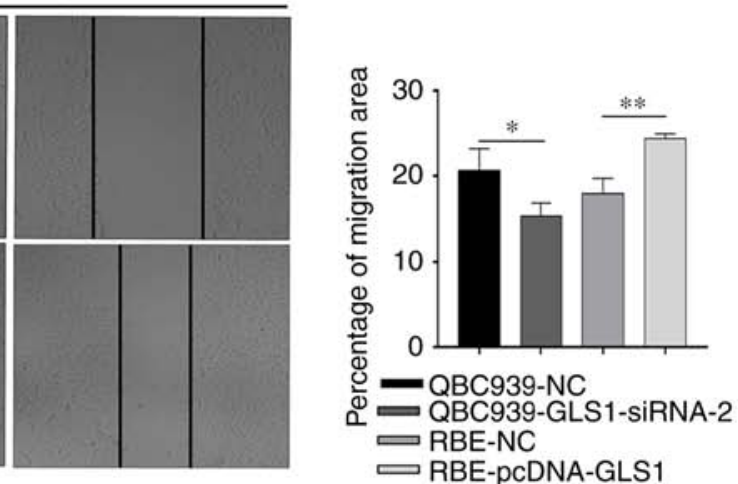

Figure 2. GLS1 regulates invasion and migration in QBC939 and RBE cells. (A) Western blot analysis was used to analyze the expression of GLS1 in ICC cell lines. (B and C) ICC cells were transfected with siRNA/pcDNA, and western blot analysis was performed. (D and E) Effects of GLS1 expression on cell invasion and migration were examined by (D) a Transwell assay and (E) a wound-healing assay (E). ${ }^{*} \mathrm{P}<0.05,{ }^{* * *} \mathrm{P}<0.01$ and ${ }^{* * * *} \mathrm{P}<0.001$. GLS1, kidney-type glutaminase; ICC, intrahepatic cholangiocarcinoma.

\section{Results}

GLS1 is overexpressed in ICC tumor specimens. First, data from the Oncomine database were used to analyze GLS1 transcripts in 3 types of digestive system tumors. As shown in Fig. 1A, the mRNA levels of GLS1 in the tumor tissues samples were higher than those in the paired peritumoral tissues samples, including in the liver cancer, colorectal cancer and gastric cancer samples (14-16). However, the expression of GLS1 in ICC tissues remains unclear. Subsequently, the GLS1 protein levels in the ICC tissue samples and matched peritumoral specimens were examined by western blot analysis and immunohistochemistry. The results revealed that the GLS1 protein levels were higher in the ICC tumor tissue samples than in the adjacent normal tissue samples $(\mathrm{P}<0.001$; Fig. 1B and $\mathrm{D})$ and found that GLS1 was mainly expressed in the cytoplasm (Fig. 1C). 
A

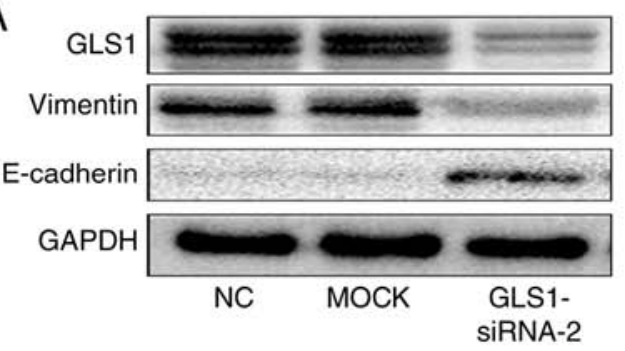

B

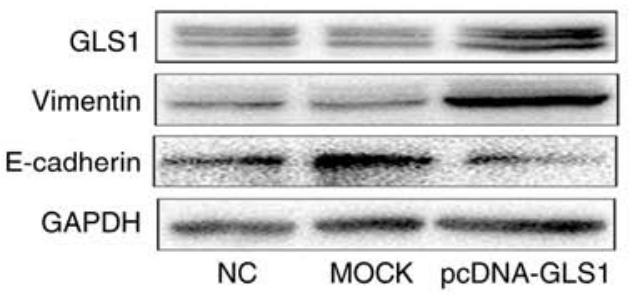

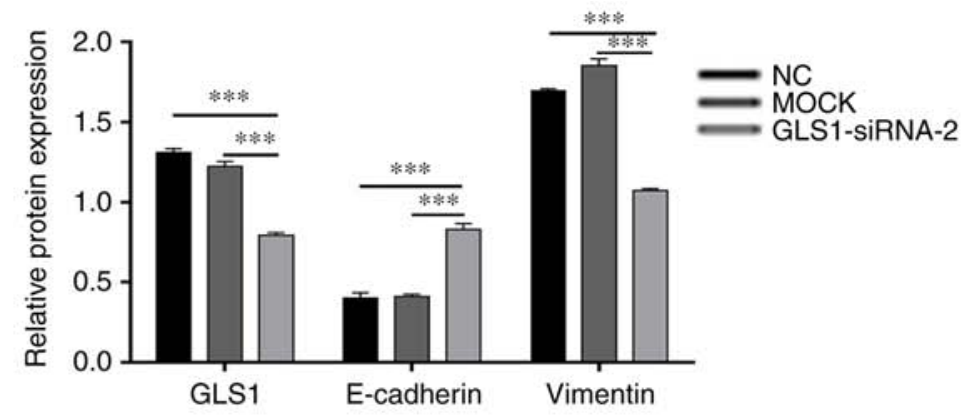

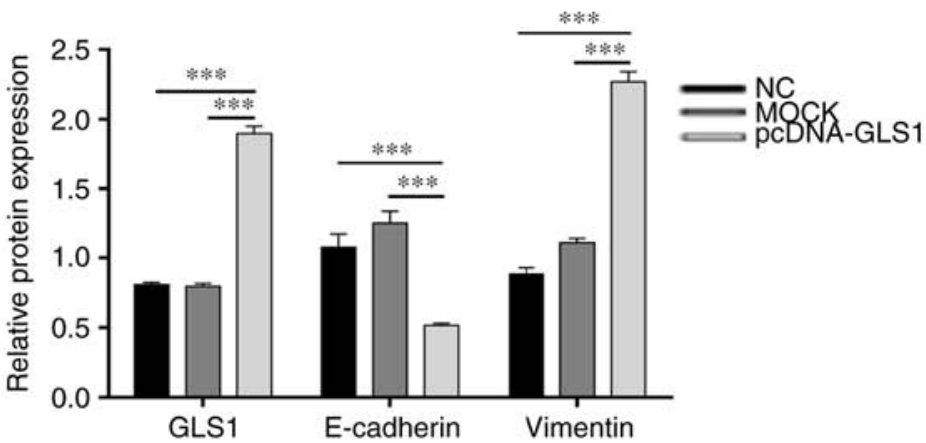

C
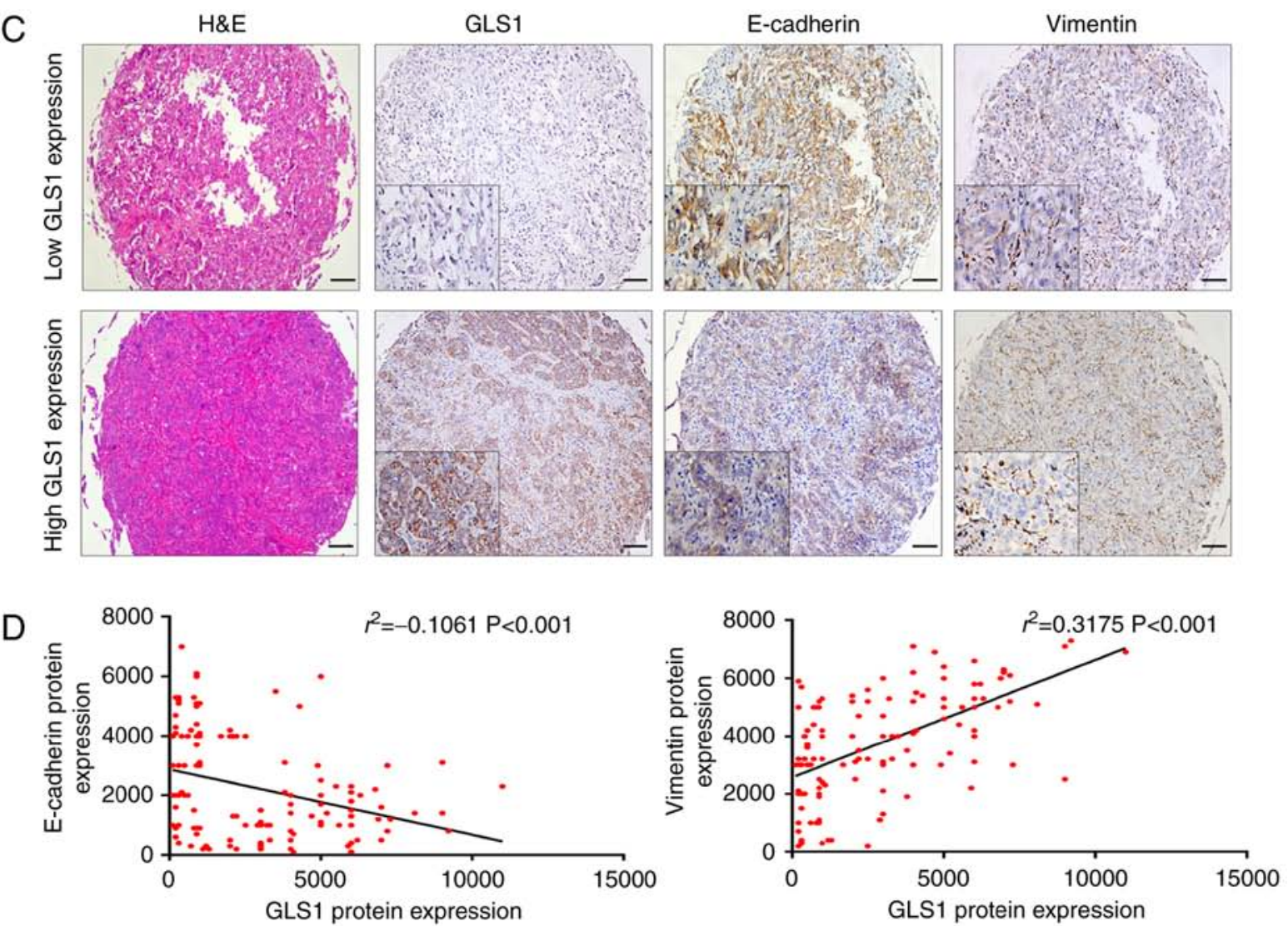

Figure 3. GLS1 successfully regulates EMT-related markers in QBC939 and RBE cells. (A and B) GLS1 regulated EMT-associated protein expression according to the results of western blot analysis. (C) Representative images of a sample from a patient with ICC that exhibited a GLS1 ${ }^{\text {high }}$, Vimentin ${ }^{\text {high }}$ and E-cadherin ${ }^{\text {low }}$ phenotype and a sample from a patient who exhibited a GLS1 ${ }^{\text {low }}$, Vimentin ${ }^{\text {low }}$ and E-cadherin ${ }^{\text {high }}$ phenotype. Scale bars, $100 \mu \mathrm{m}$. (D) GLS1 expression negatively correlated with E-cadherin expression and positively associ correlated ated with Vimentin expression. ${ }^{* * *} \mathrm{P}<0.001$. GLS1, kidney-type glutaminase; ICC, intrahepatic cholangiocarcinoma.

GLS1 regulates the migratory and invasive abilities of ICC cells. To further examine the role of GLS1 in ICC cell invasion and migration, the GLS1 protein levels were examined in a panel of ICC cell lines (QBC939, HCCC-9810 and RBE) by western blot analysis (Fig. 2A). The data indicated that the QBC939 cells exhibited the highest expression of GLS1 and that the RBE cells exhibited the lowest expression level of GLS1 among all the ICC cell lines examined. Therefore, 
A

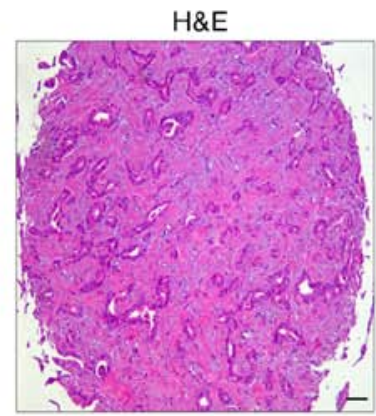

C

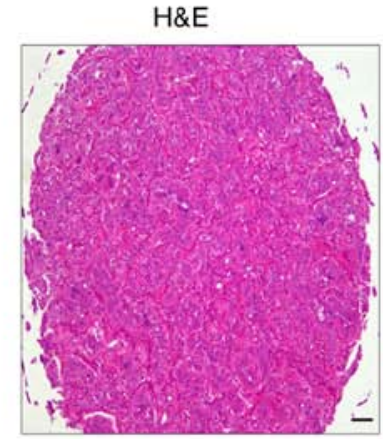

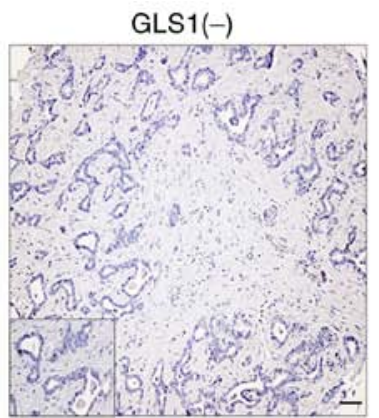

B
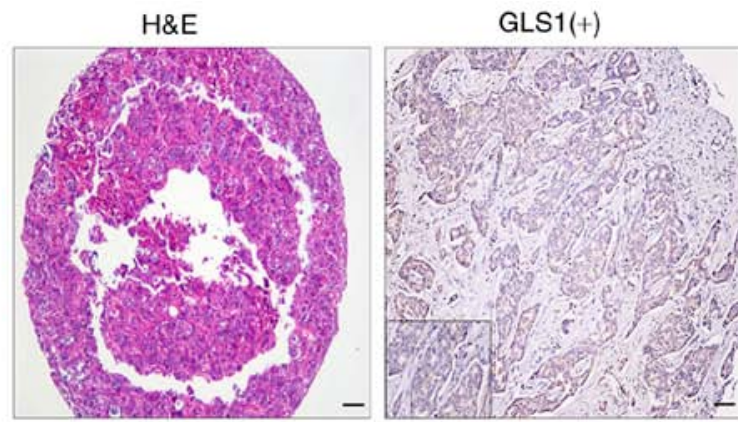

D

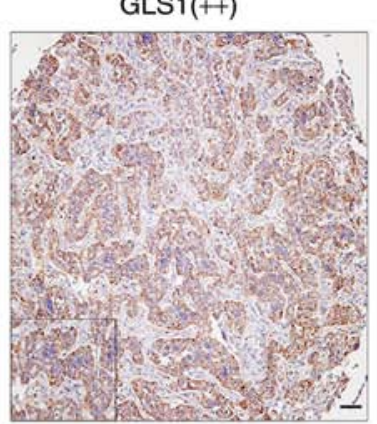

H\&E

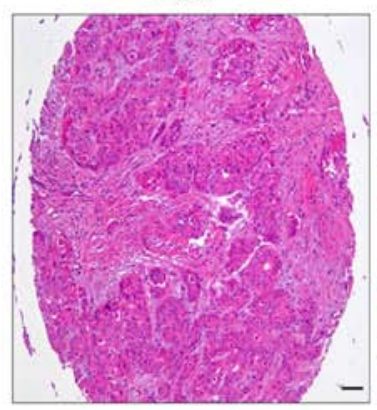

GLS1(+++)

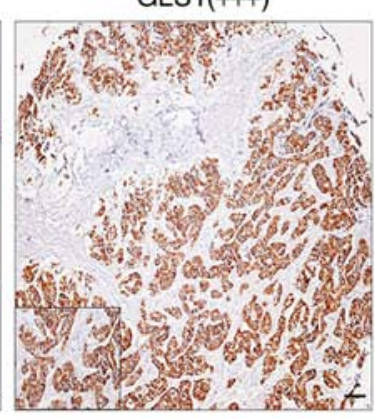

E

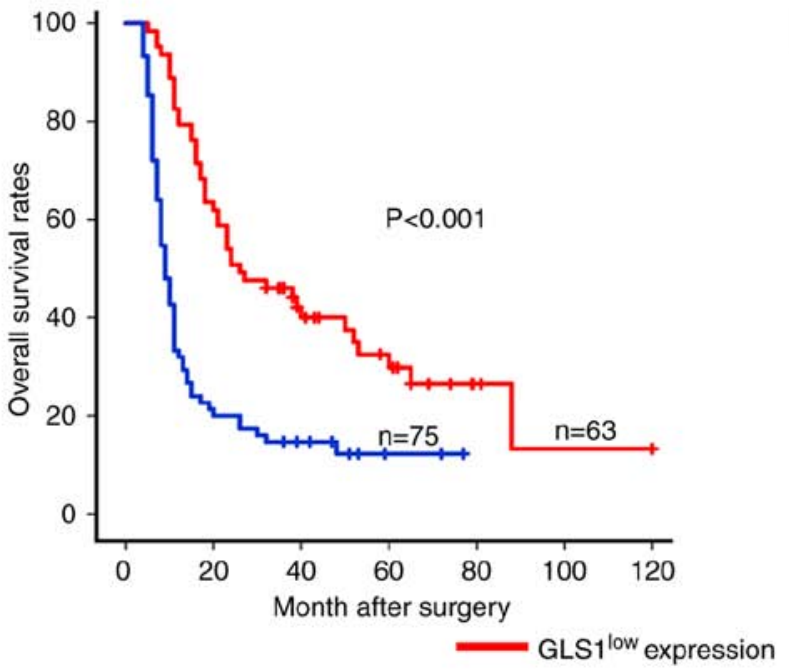

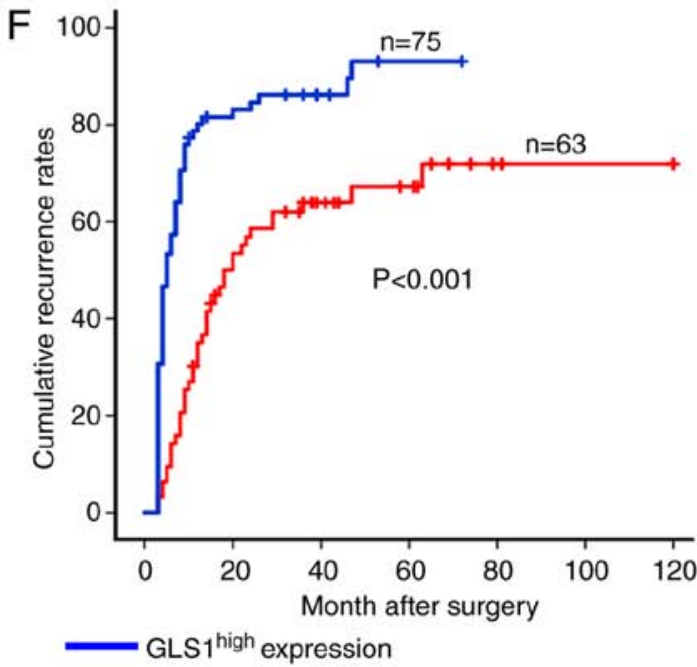

Figure 4. Log-rank tests and Kaplan-Meier survival curves of patients with ICC stratified according to their GLS1 protein expression. (A-D) Representative images of H\&E and immunohistochemical staining for GLS1 expression. (A) Negative; (B) weak; (C) moderate; and (D) intensive. (E) OS curves stratified by GLS1 expression. (F) Cumulative recurrence rate curves stratified by GLS1 expression. Scale bars, $100 \mu \mathrm{m}$. GLS1, kidney-type glutaminase; ICC, intrahepatic cholangiocarcinoma; OS, overall survival.

the QBC939 cell line and RBE cell line were used in the subsequent experiments. The results of western blot analysis revealed that GLS1 expression was markedly knocked down in the GLS1-siRNA-2-transfected cell lines (Fig. 2B). Therefore, GLS1-siRNA-2 was used in the subsequent experiments. GLS1 was also overexpressed in the RBE cells (Fig. 1C). Subsequently, a Transwell assay revealed that the GLS1-siRNA-2-transfected cells exhibited a significantly lower rate of invasion than the GLS1-NC cells (Fig. 2D). A wound-healing assay also indicated that the downregulation of GLS1 decreased migration of the GLS1-siRNA-2 ICC cells compared with that of the GLS1-NC cells at $24 \mathrm{~h}$ (Fig. 2E). However, compared with the NC cells, the RBE cells with an upregulated GLS1 expression exhibited increased invasion and metastasis (Fig. 2D and E). These data indicate that
GLS1 positively regulates the migratory and invasive abilities of ICC cells.

GLS1 regulates the levels of EMT-related markers in ICC cells. Recently, a previous study demonstrated that GLS1 reduces cell-cell contact and increases cell motility by inducing EMT in lung cancer cells (12). Thus, in this study, the expression of two EMT-related markers, Vimentin and E-cadherin, was determined by western blot analysis. This experiment indicated that the expression of Vimentin was significantly reduced, whereas the levels of E-cadherin were significantly increased following the knockdown of GLS1 expression in QBC939 cells ( $\mathrm{P}<0.001$; Fig. 3A). However, the overexpression of GLS1 in the RBE cells yielded opposite results ( $\mathrm{P}<0.001$; Fig. 3B). Subsequently, using an ICC tissue 
Table II. Univariate and multivariate analyses of factors associated with recurrence and survival of patients with ICC.

\begin{tabular}{|c|c|c|c|c|c|c|}
\hline \multirow[b]{3}{*}{ Variable } & \multicolumn{3}{|c|}{ Overall survival } & \multicolumn{3}{|c|}{ Cumulative recurrence } \\
\hline & \multirow{2}{*}{$\frac{\text { Univariate }}{\text { P-value }}$} & \multicolumn{2}{|c|}{ Multivariate } & \multirow{2}{*}{$\frac{\text { Univariate }}{\text { P-value }}$} & \multicolumn{2}{|c|}{ Multivariate } \\
\hline & & HR (95\% CI) & P-value & & HR $(95 \%$ CI $)$ & P-value \\
\hline Sex (male vs. female) & 0.847 & & NA & 0.921 & & NA \\
\hline Age, years ( $\geq 53$ vs. $<53$ ) & 0.491 & & NA & 0.338 & & NA \\
\hline HBsAg (positive vs negative) & 0.949 & & NA & 0.518 & & NA \\
\hline Child-Pugh score (a vs. B) & 0.131 & & NA & 0.388 & & NA \\
\hline Serum CA 19-9, ng/ml ( $\geq 37$ vs., $<37)$ & 0.272 & & NA & 0.215 & & NA \\
\hline Serum ALT, U/l ( $\geq 75$ vs., $<75$ ) & 0.599 & & NA & 0.799 & & NA \\
\hline AFP & 0.130 & & NA & 0.562 & & NA \\
\hline CEA & 0.981 & & NA & 0.361 & & NA \\
\hline GGT & 0.044 & & NS & 0.190 & & NA \\
\hline Cirrhosis (yes vs. no) & 0.276 & & NA & 0.458 & & NA \\
\hline Tumor size (diameter, $\mathrm{cm})(\geq 5$ vs., $<5)$ & 0.022 & $\begin{array}{c}0.562 \\
(0.338-0.935)\end{array}$ & 0.026 & 0.005 & $\begin{array}{c}0.540 \\
(0.282-0.816)\end{array}$ & 0.007 \\
\hline Tumor number (multiple vs. solitary) & 0.007 & & NS & 0.001 & $\begin{array}{c}0.479 \\
(0.235-0.882)\end{array}$ & 0.020 \\
\hline Embolus (yes vs. no) & 0.032 & & NS & 0.031 & $\begin{array}{c}0.619 \\
(0.403-0.952)\end{array}$ & 0.290 \\
\hline Capsulation (yes vs. no) & 0.252 & & NA & 0.125 & & NA \\
\hline Lymphatic metastasis (yes vs. no) & 0.007 & $\begin{array}{c}0.539 \\
(0.352-0.826)\end{array}$ & 0.040 & 0.001 & $\begin{array}{c}0.619 \\
(0.352-0.826)\end{array}$ & 0.029 \\
\hline Tumor differentiation (III/IV vs. I/II) & 0.069 & & NS & 0.059 & & NA \\
\hline GLS1 density $(<50 \%$ vs. $\geq 50 \%)$ & $<0.001$ & $\begin{array}{c}2.718 \\
(1.820-4.059)\end{array}$ & $<0.001$ & $<0.001$ & $\begin{array}{c}2.774 \\
(1.84-4.182)\end{array}$ & $<0.001$ \\
\hline
\end{tabular}

AFP, $\alpha$-fetoprotein; ALT, alanine aminotransferase; CA19-9, carbohydrate antigen 19-9; HBsag, hepatitis B surface antigen; GGT, $\gamma$-glutamyl transferase; ICC, intrahepatic cholangiocarcinoma; GLS1, Kidney-type glutaminase; NS, not significant; NA, not available .

array, immunohistochemistry was performed to simultaneously analyze GLS1, Vimentin and E-cadherin expression (Fig. 3C). The results indicated that in the human ICC tissue, the GLS1 levels were positively associated with Vimentin expression and negatively associated with E-cadherin expression. In addition, Spearman's correlation analysis also revealed that the GLS1 levels positively correlated with Vimentin expression $\left(\mathrm{r}^{2}=0.3175 ; \mathrm{P}<0.001\right)$ and negatively correlated with E-cadherin expression $\left(r^{2}=-0.1061 ; \mathrm{P}<0.001\right.$; Fig. 3D). These data indicate that the expression of GLS1 is essential for the EMT process and for the progression of ICC.

A high expression of GLS1 is associated with a poor prognostic phenotype. The samples were classified into 2 groups, a GLS1 ${ }^{\text {high }}(++$, moderate; +++ , strong) group and a GLS1 ${ }^{\text {low }}$ (-, absent; +, weak) group, according to the mean value of the expression of GLS1 in the tumor tissue samples (Fig. 4A-D). The GLS1 ${ }^{\text {high }}$ group accounted for $54.3 \%(n=75)$ of the samples, and the GLS1 ${ }^{\text {low }}$ group accounted for $45.7 \%$ $(n=63)$. We found that the overexpression of GLS1 was associated with malignant phenotypic features, such as lymphatic metastasis $(\mathrm{P}=0.029)$ and poor tumor differentiation $(\mathrm{P}=0.001)$ (Table I). By contrast, other clinicopathological characteristics including age, microvascular invasion, tumor size and number were not associated with GLS1 expression. The GLS1 $1^{\text {high }}$ expression group was also found to be associated with a worse OS time than the GLS1 ${ }^{\text {low }}$ expression group $(\mathrm{P}<0.001$; Fig. 4E). The 2- and 5-year OS rates in the GLS1 ${ }^{\text {high }}$ group were significantly lower than those in the GLS1 ${ }^{\text {low }}$ group (21.1 vs. $53.3 \%$ and 14.5 vs. $35.5 \%$, respectively). The $2-$ and 5 -year cumulative recurrence rates were also markedly higher in the GLS $1^{\text {high }}$ group compared with the GLS1 ${ }^{\text {low }}$ group (81.5 vs. $55.5 \%$ and 88.1 vs. $66.1 \%$, respectively; Fig. 4E). Univariate analysis revealed that GLS1 expression, tumor size, tumor number, embolus and lymphatic metastasis were significantly associated with OS and cumulative recurrence rate in patients with ICC (Table II). In addition, multivariate analysis revealed that GLS1 expression was an independent predictor of OS $(\mathrm{P}<0.001)$ and cumulative recurrence $(\mathrm{P}<0.001)$ (Table II).

\section{Discussion}

In this study, we demonstrated that GLS1 was overexpressed in ICC tissue compared with adjacent normal tissue, and the downregulation of GLS1 expression in QBC939 cells suppressed ICC cell invasion and migration. The expression of the EMT mesenchymal marker, Vimentin, was downregulated following the knockdown of GLS1 expression in QBC939 cells. 
By contrast, the expression of the epithelial marker, E-cadherin, was upregulated. However, the overexpression of GLS1 in the RBE cells induced a lower expression of E-cadherin and a higher expression of Vimentin. Clinically, we detected GLS1 expression among 138 patients with ICC. The results revealed that a high GLS1 expression was strongly associated with poor tumor differentiation, lymphatic metastasis, early recurrence and an unfavorable prognosis. Patients with a high expression of GLS1 had a poorer OS and higher cumulative recurrence rates than patients with a low GLS1 expression.

The 'Warburg effect' describes the phenomenon of cancer cells creating energy predominantly from the glycolytic breakdown of glycose, rather than mitochondrial oxidative phosphorylation (17). In most situations, cancer cells that exhibit the Warburg effect also exhibit a significant dependence on glutamine and cannot proliferate in cell culture without this molecule, a state called 'glutamine addiction' (18). GLS1 dysregulation has been reported in a number of types of cancer. For instance, Pan et al (19) found that GLS1 participated in the TCA cycle, elevating glucose intake and promoting the growth of prostate cancer cells. In our previous study, we also found that GLS1 protein was frequently expressed in HCC tissue samples and that this expression was associated with a poor prognosis (7). The underlying mechanisms may be associated with the EMT process. Research has indicated that GLS1 regulates E-cadherin and Snail in MCF-7 cells (12), which indicates that GLS1 can regulate EMT-associated genes. Recent evidence has also indicated that GLS1 plays a significant role in the progression of ICC, suggesting that GLS1 may be a novel prognostic factor and treatment target in ICC.

Previous studies have reported that EMT is a potential mechanism of cancer metastasis, and this process activates the mesenchymal phenotype and represses the epithelial phenotype, driving separation from the primary tumor (20-22). Recently, GLS1 was demonstrated to regulate Vimentin, E-cadherin and Snail expression $(12,23)$. Therefore, GLS1 may regulate E-cadherin and Vimentin expression in ICC. The findings of this study indicated that GLS1 expression negatively correlated with E-cadherin expression and positively correlated with Vimentin expression. Moreover, the regulation of GLS1 expression affected E-cadherin and Vimentin expression in ICC cells. These data suggest that there is a potential association between GLS1 and EMT as regards the progression of ICC.

In conclusion, the interactions of GLS1 with E-cadherin and Vimentin were confirmed in this study. However, whether other EMT markers are regulated by GLS1 warrants further investigation in the future. The results of this study, suggest that GLS1 may prove to be an innovative therapeutic target in patients with ICC.

\section{Acknowledgements}

Not applicable.

\section{Funding}

This study was supported by the Health research project in Jiangsu Province (grant no. H201661), Cancer Biology State Key
Laboratory Project (grant no. CBSKL201717) and the National Natural Science Foundation of China (grant no. 81702861).

\section{Availability of data and materials}

The datasets used and/or analyzed during the current study are available from the corresponding author upon reasonable request.

\section{Authors' contributions}

JC and DSB were involved in study design and drafted the manuscript. CZ, GQJ, SJJ and AWK were involved in TMA analysis. ZHG, DCY and QW were involved in statistical analysis. YQF, DWL and AQW were involved in clinical data collection. DSB was involved in the study design, financial support and proof-reading of the manuscript. All authors read and approved the final manuscript.

\section{Ethics approval and consent to participate}

The use of these tissue specimens was approved by the Zhongshan Hospital Research Ethics Committee and written consent was obtained from the patients.

\section{Patient consent for publication}

Not applicable.

\section{Competing interests}

The authors declare that they have no competing interests.

\section{References}

1. Bartella I and Dufour JF: Clinical diagnosis and staging of intrahepatic cholangiocarcinoma. J Gastrointestin Liver Dis 24: 481-489, 2015.

2. Guo LH and $\mathrm{Xu} \mathrm{HX:} \mathrm{Contrast-enhanced} \mathrm{ultrasound} \mathrm{in} \mathrm{the}$ diagnosis of hepatocellular carcinoma and intrahepatic cholangiocarcinoma: Controversy over the ASSLD Guideline. Biomed Res Int 2015: 349172, 2015.

3. Guro H, Kim JW, Choi Y, Cho JY, Yoon YS and Han HS: Multidisciplinary management of intrahepatic cholangiocarcinoma: Current approaches. Surg Oncol 26: 146-152, 2017.

4. Yoh T, Hatano E, Nishio T, Seo S, Taura K, Yasuchika K, Okajima H, Kaido T and Uemoto S: Significant improvement in outcomes of patients with intrahepatic cholangiocarcinoma after surgery. World J Surg 40: 2229-2236, 2016.

5. Guglielmi A, Ruzzenente A, Campagnaro T, Pachera S, Valdegamberi A, Nicoli P, Cappellani A, Malfermoni G and Iacono C: Intrahepatic cholangiocarcinoma: Prognostic factors after surgical resection. World J Surg 33: 1247-1254, 2009.

6. Hanahan D and Weinberg RA: Hallmarks of cancer: The next generation. Cell 144: 646-674, 2011.

7. Yu D, Shi X, Meng G, Chen J, Yan C, Jiang Y, Wei J and Ding Y: Kidney-type glutaminase (GLS1) is a biomarker for pathologic diagnosis and prognosis of hepatocellular carcinoma. Oncotarget 6: 7619-7631, 2015.

8. Qie S, Chu C, Li W, Wang C and Sang N: ErbB2 activation upregulates glutaminase 1 expression which promotes breast cancer cell proliferation. J Cell Biochem 115: 498-509, 2014.

9. Huang F, Zhang Q, Ma H, Lv Q and Zhang T: Expression of glutaminase is upregulated in colorectal cancer and of clinical significance. Int J Clin Exp Pathol 7: 1093-1100, 2014.

10. Huang F, Wang BR and Wang YG: Role of autophagy in tumorigenesis, metastasis, targeted therapy and drug resistance of hepatocellular carcinoma. World J Gastroenterol 24: 4643-4651, 2018. 
11. Klymkowsky MW: Beta-catenin and its regulatory network Hum Pathol 36: 225-227, 2005.

12. Lee SY, Jeon HM, Ju MK, Jeong EK, Kim CH, Park HG, Han SI and Kang HS: Dlx-2 and glutaminase upregulate epithelial-mesenchymal transition and glycolytic switch. Oncotarget 7: 7925-7939, 2016.

13. Bai DS, Dai Z, Zhou J, Liu YK, Qiu SJ, Tan CJ, Shi YH, Huang C, Wang Z, He YF and Fan J: Capn4 overexpression underlies tumor invasion and metastasis after liver transplantation for hepatocellular carcinoma. Hepatology 49: 460-470, 2009.

14. Roessler S, Jia HL, Budhu A, Forgues M, Ye QH, Lee JS, Thorgeirsson SS, Sun Z, Tang ZY, Qin LX and Wang XW: A unique metastasis gene signature enables prediction of tumor relapse in early-stage hepatocellular carcinoma patients. Cancer Res 70: 10202-10212, 2010.

15. Cui J, Chen Y, Chou WC, Sun L, Chen L, Suo J, Ni Z, Zhang M, Kong X, Hoffman LL, et al: An integrated transcriptomic and computational analysis for biomarker identification in gastric cancer. Nucleic Acids Res 39: 1197-1207, 2011.

16. Skrzypczak M, Goryca K, Rubel T, Paziewska A, Mikula M, Jarosz D, Pachlewski J, Oledzki J and Ostrowski J: Modeling oncogenic signaling in colon tumors by multidirectional analyses of microarray data directed for maximization of analytical reliability. PLoS One 5: pii: e13091, 2010.

17. Zhao Y, Butler EB and Tan M: Targeting cellular metabolism to improve cancer therapeutics. Cell Death Dis 4: e532, 2013.
18. Abu Aboud O, Habib SL, Trott J, Stewart B, Liang S, Chaudhari AJ, Sutcliffe J and Weiss RH: Glutamine addiction in kidney cancer suppresses oxidative stress and can Be exploited for real-time imaging. Cancer Res 77: 6746-6758, 2017.

19. Pan T, Gao L, Wu G, Shen G, Xie S, Wen H, Yang J, Zhou Y, Tu Z and Qian W: Elevated expression of glutaminase confers glucose utilization via glutaminolysis in prostate cancer. Biochem Biophys Res Commun 456: 452-458, 2015.

20. Krebs AM, Mitschke J, Lasierra Losada M, Schmalhofer O, Boerries M, Busch H, Boettcher M, Mougiakakos D, Reichardt W, Bronsert P, et al: The EMT-activator Zeb1 is a key factor for cell plasticity and promotes metastasis in pancreatic cancer. Nat Cell Biol 19: 518-529, 2017.

21. Qureshi R, Arora H and Rizvi MA: EMT in cervical cancer: Its role in tumour progression and response to therapy. Cancer Lett 356: 321-331, 2015.

22. Terry S, Savagner P, Ortiz-Cuaran S, Mahjoubi L, Saintigny P, Thiery JP and Chouaib S: New insights into the role of EMT in tumor immune escape. Mol Oncol 11: 824-846, 2017.

23. Ulanet DB, Couto K, Jha A, Choe S, Wang A, Woo HK, Steadman M, DeLaBarre B, Gross S, Driggers E, et al: Mesenchymal phenotype predisposes lung cancer cells to impaired proliferation and redox stress in response to glutaminase inhibition. PLoS One 9: e115144, 2014. 